\title{
Criminologie
}

\section{Presse et corps policiers : complicité et conflit}

\section{Georges-André Parent}

Volume 20, numéro 1, 1987

Faire les nouvelles : journalisme et affaires criminelles

URI : https://id.erudit.org/iderudit/017248ar

DOI : https://doi.org/10.7202/017248ar

Aller au sommaire du numéro

Éditeur(s)

Les Presses de l'Université de Montréal

ISSN

0316-0041 (imprimé)

1492-1367 (numérique)

Découvrir la revue

\section{Citer cet article}

Parent, G.-A. (1987). Presse et corps policiers : complicité et conflit. Criminologie, 20(1), 99-120. https://doi.org/10.7202/017248ar

\section{Résumé de l'article}

The literature abounds with studies showing the cultural gap and the hostility that exists between journalists and the police. During the 19th century in the United States, however, a complicity eminently profitable for both was rapidly established between constables and reporters for the first penny newspapers. The confrontations and mass rallies of the 60 ' s saw the role of journalists change to become no longer the servile and docile distributors of a particular image of crime, the criminal and police work. Journalists suddenly found themselves on the side of the "criminals", facing the truncheons of militant police.

In Montreal, a public relations service was subsequently created to restore the positive image of the police and try to reestablish the control of information. Since the newspapers were more commercial than intellectual enterprises, complicity, both official and unofficial, was quickly reestablished, giving rise to a rather doubtful relationship between journalists and the police.

It was about ten years after the October crisis, when the majority of journalists identified more with the protesters than with the repressive forces, that the Quebec Police decided to restore media/police relationships to their former state. A communications service was created, which, in little more than ten years, enabled the police authorities to exercise an almost total control over information; only what served the strategy of the police was to be published.

For the R.C.M.P., the honeymoon came to an end with the creation of the Keable and McDonald Commissions. In 1977, there were five policemen attached to the public relations service of the R.C.M.P. in Montreal. In 1986, a single officer remains and no longer even bears the title of official communications or public relations officer.

Everywhere in Quebec, journalists seem to have traded their ability to inform for their daily ration of diverse facts, and it is still the disturbing image of crime and criminals that they blithely publish, making the media true instruments of social control.
Ce document est protégé par la loi sur le droit d'auteur. L'utilisation des services d’Érudit (y compris la reproduction) est assujettie à sa politique d'utilisation que vous pouvez consulter en ligne.

https://apropos.erudit.org/fr/usagers/politique-dutilisation/ 
The literature abounds with studies showing the cultural gap and the hostility that exists between journalists and the police. During the 19th century in the United States, however, a complicity eminently profitable for both was rapidly established between constables and reporters for the first penny newspapers.

The confrontations and mass rallies of the 60's saw the role of journalists change to become no longer the servile and docile distributors of a particular image of crime, the criminal and police work. Journalists suddenly found themselves on the side of the "criminals", facing the truncheons of militant police.

In Montreal, a public relations service was subsequently created to restore the positive image of the police and try to reestablish the control of information. Since the newspapers were more commercial than intellectual enterprises, complicity, both official and unofficial, was quickly reestablished, giving rise to a rather doubtful relationship between journalists and the police.

It was about ten years after the October crisis, when the majority of journalists identified more with the protesters than with the repressive forces, that the Quebec Police decided to restore medialpolice relationships to their former state. A communications service was created, which, in little more than ten years, enabled the police authorities to exercise an almost total control over information; only what served the strategy of the police was to be published.

For the R.C.M.P., the honeymoon came to an end with the creation of the Keable and McDonald Commissions. In 1977, there were five policemen attached to the public relations service of the R.C.M.P. in Montreal. In 1986, a single officer remains and no longer even bears the title of official communications or public relations officer.

Everywhere in Quebec, journalists seem to have traded their ability to inform for their daily ration of diverse facts, and it is still the disturbing image of crime and criminals that they blithely publish, making the media true instruments of social control.

\section{INTRODUCTION}

Étrange paradoxe que cette histoire des relations police-médias, de ces cent ans d'interrelations à la fois faites d'hostilité et de complicité!

* Georges-André Parent, journaliste dans le domaine policier et judiciaire depuis 25 ans; directeur du journal Allo-Police pendant 10 ans; présentement étudiant en criminologie. 
Méfiance, hostilité, mépris ne sont que quelques aspects de ce vieil antagonisme. Leurs rôles (du moins ceux qu'ils se donnent), leurs informations, leur travail quotidien, leur appartenance à des cultures différentes auraient dû faire des policiers et des journalistes deux groupes en perpétuel conflit. Altschull (1975), Selke et Bartoszek (1984) ont bien analysé ce phénomène.

Pourtant, aussi enraciné soit-il, ce conflit ne résiste pas à l'interaction immédiate et concrète des deux groupes qui en arrivent à une connivence, une collusion qui font de l'un l'auxiliaire de l'autre. La relation médias-police est d'abord et avant tout la légitimation de l'action policière et de son utilité, de même que la diffusion d'une certaine image du crime, du criminel et des policiers. Cent cinquante ans plus tard, c'est encore le constable volontaire qui doit justifier son institution et son action en projetant l'image d'une société dangereuse et du policier chasseur de criminels et protecteur du citoyen menacé. Les médias deviennent son grand complice et se chargent de diffuser cette image éminemment rentable pour eux qui sont passés des pamphlets socio-politiques aux "penny journal» et aux médias de masse, des entreprises plus commerciales qu'intellectuelles. La littérature foisonne d'études sur le sujet: Davis (1952); Fishman (1978); Gosselin, McFadden, Pilon et Touchette (1978); André (1979); Humphries (1981); Poveda (1982); Christiensen, Schmidt et Henderson (1982); Smaus (1983), pour n'en nommer que quelques-unes.

Plus les policiers se sont faits les croisés de la lutte au crime, plus les journalistes ont découvert là un merveilleux filon, une matière première idéale pour la vente des journaux. Plus les journaux ont couvert le crime, plus les policiers ont pu obtenir d'effectifs et de pouvoir... et plus les policiers sont devenus nombreux et puissants, plus ils ont pu produire de crimes... et plus les lecteurs de journaux ont craint le crime, plus on a pu vendre de journaux... Cent ans plus tard, cette mécanique n'est pas encore usée!

Les policiers se concentrent sur certaines cibles faciles et certaines formes d'illégalismes que les médias diffusent parce que leurs clients en demandent... et plus le client en demande, plus les médias et la police en produisent... On vend la peur du crime comme les producteurs de cinéma vendent des thrillers. Sauf qu'ici, Rocky, Rambo et les autres ne sont pas que porteurs d'images. Les médias d'information deviennent ainsi, à la presque solde des policiers, d'extraordinaires instruments de contrôle social et de répression. 
C'est cette interaction que nous tenterons d'illustrer dans cet article et de traduire dans la réalité concrète, particulièrement à Montréal, au risque de sembler faire parfois de l'anecdote plus que de l'analyse.

Jusqu'aux années 60, à Montréal comme partout en Amérique, une espèce de compromis, d'arrangement tacite, a réussi à concilier les intérêts de l'un et l'autre. Chaque journal avait "son" reporter policier attitré et chaque corps de police avait "ses" journalistes. Les policiers foumissaient donc de la "bonne copie" tous les jours à leurs journalistes. pourvu que ceux-ci se contentent des versions policières officielles et demeurent des auxiliaires condescendants et pas trop critiques de leurs pourvoyeurs de nouvelles. La complicité du journaliste leur était acquise. Le chroniqueur de police, généralement un vieux journaliste sans grand avenir, qui ne rêvait déjà plus de devenir un jour éditorialiste ou rédacteur en chef, avait tout intérêt à rester collé aux policiers. C'était sa garantie de foumir de la «bonne copie» tous les jours à son chef de nouvelles, d'être le seul à pouvoir obtenir des scoops, de pouvoir avoir la «vraie» version de tel meurtre ou incendie spectaculaires. Tous les journaux n 'étaient pas alors à sensations mais aucun quotidien ne se privait du fait divers. Les quotidiens du matin sont vite devenus plus sensationnels, avec leurs trois ou quatre premières pages à peu près consacrées exclusivement au fait divers. Les journaux d'après-midi donnaient moins d'importance à ce genre de nouvelles mais on les trouvait toutes, en condensé, dans les colonnes des journaux les plus respectables.

Il arrivait bien parfois qu'on dénonce certaines actions policières. Ce pouvait être le chroniqueur politique, rapportant les audiences d'une commission d'enquête; le chroniqueur syndical, dénonçant la violence policière sur les lignes de piquetage; le chroniqueur judiciaire, rapportant la thèse de la défense plutôt que celle des policiers. D'une façon générale, c'est le pouvoir politique qu'on attaquait à travers les policiers. Un Jacques Hébert s'est retrouvé en prison davantage pour s'être attaqué au Premier ministre Duplessis qu’à l'enquête policière menée dans l'affaire Coffin.

Grâce à leurs journalistes, les policiers pouvaient neutraliser ces campagnes, créer des diversions, pratiquer allègrement la désinformation. Rien n'a bien changé à ce chapitre et nous verrons quelques récents exemples de telles manœuvres.

Le chroniqueur policier ne se brûlait donc pas, laissant à d'autres le soin d'écorcher de temps à autre la belle et bonne image des policiers. Les policiers, eux, gardaient intact leur contact à l'intérieur du journal, malgré les éditorialistes-défenseurs-des-droits-et-libertés et autres chro- 
niqueurs aux idées plus libérales pour qui le policier est et ne peut être que l'instrument répressif du pouvoir politique. Les policiers sont, d'ailleurs, très sélectifs dans leur choix de journalistes. Ils ont tôt fait d'éliminer un jeune journaliste trop curieux, indépendant ou ambitieux, en le privant littéralement de nouvelles. Les journalistes ne moisissent généralement pas longtemps aux faits divers et ne voient dans cette assignation qu'une étape dans leur carrière. Jusqu'aux années 60 , d'ailleurs, on disait encore de la chronique des faits divers et policiers que c'était encore la meilleure école de journalisme. Pendant que de jeunes loups y faisaient leurs classes, on laissait le bon vieux chroniqueur attitré faire son job, bien à l'abri des regards indiscrets, en compagnie de ses amis policiers.

Les années 60 sont venues briser cet échange de bons procédés. Plusieurs auteurs américains y voient là le début de la concrétisation de cette hostilité jusque-là surtout théorique entre policiers et médias (Poveda, 1982; Selke et Bartoszek, 1984; Alschull, 1975; entre autres). Pendant qu'on remettait en question le FBI de J.-Edgar Hoover aux USA, on dénonçait enfin chez nous la police du régime Duplessis.

Les journaux ont continué, encore plus que jamais, à exploiter le fait divers et la couverture des crimes majeurs, surtout de violence, mais une nouvelle source de nouvelles s'est présentée: les manifestations. Manifestations de Noirs, d'étudiants, de contestataires de toutes étiquettes. Aux États Unis, ce fut Kennedy, le Viet-Nam, le peace and love, la ségrégation; au Québec: les premiers grands conflits dans la Fonction publique, la contestation étudiante, les manifestations nationalistes, la première vague felquiste, la multiplication des grèves.

Au même moment, les médias électroniques ont fait leur entrée dans le décor, fracassant toutes les petites règles non écrites, ces petites ententes tacites qui avaient défini jusque-là les relations police-journalistes. Dans ce domaine la concurrence était sauvage. C'était plus que jamais la course au scoop. On ne pouvait plus attendre le traditionnel pourvoyeur de nouvelles, avec ses précieux contacts. Peu importait, dorénavant, d'avoir "LA VRAIE" histoire mais bien plutôt la première image, d'être les premiers sur les lieux.

Stations de télévision et de radio se sont mises à équiper leurs jeunes journalistes de voitures à la James Bond, dotées de scanners, de longues antennes et de girophares, qui se précipitaient sur les lieux des accidents, des incendies et des meurtres, en même temps que les policiers. Être sur les lieux, c'était le nouveau mot d'ordre. On ne s'embarrassait plus d'obtenir la version policière ou la permission de ces messieurs pour interviewer un témoin ou une victime. Les policiers venaient de perdre 
littéralement le contrôle de l'information. Même les grands journaux ont dû emboîter le pas et entrer dans la course à la nouvelle.

Chez les journalistes, au même moment, une sympathie naturelle s'établissait avec les divers contestataires. Les journalistes retrouvaient l'euphorie de la grande mission de chien de garde, de la «vocation". Au même moment, les caméras de télévision, avec cette lunette déformante qu'est le zoom, projetaient au télé-journal des images de policiers matraquant de jeunes contestataires. Des journalistes ont aussi été matraqués, arrêtés. C'était la guerre ouverte. Les journaux du matin, pour affronter la concurrence de la télévision, publiaient plus de photos que de textes. Le policier-matraqueur faisait de plus en plus souvent la une des journaux du matin, alors que les grands journaux d'après-midi "éditorialisaient" sur les droits et libertés face aux forces répressives. Au Québec le sentiment nationaliste a servi de véritable catalyseur au sentiment antipolicier.

Désormais, les relations médias-police ne seraient jamais plus tout à fait les mêmes. Sur les lieux des manifestations, policiers et joumalistes devenaient concurrents. Les médias étaient souvent invités à croquer sur le vif des scénarios savamment planifiés par des manifestants. Les policiers, pris au piège, voyaient leur image démolie dans les médias. Les journalistes étaient perçus eux-mêmes par les policiers comme des provocateurs, des fomenteurs de troubles, ne recherchant systématiquement que la bavure policière, le geste violent, condamnant le policier à un rôle d'agresseur. Au Québec, de 1960 à 1970, une bonne vingtaine de journalistes (Fournier, 1982) ont été directement mêlés aux mouvements terroristes.

\section{À LA POLICE DE MONTRÉAL: RELATIONS OFFICIELLES, OFFICIEUSES ET PARTICULIÈRES...}

C'est dans ce contexte qu'a été créée en 1963 une section de relations publiques, à la police de Montréal. Il était devenu impérieux de refaire l'image de la police, d'autant plus que nous étions à l'heure des "grands ménages" de l'administration Drapeau-Saulnier en vue d'Expo 67.

Trois policiers étaient alors attachés à cette section de relations publiques. Deux, un anglophone et un francophone, pour le travail des relations publiques; un autre pour les relations auprès des jeunes. Ce demier visitait les écoles, parlait de prévention aux jeunes, essayait de leur donner une image plus positive de la police. Cette section s'est vite détachée pour devenir un service autonome. 
Les deux policiers relationnistes sont vite devenus de véritables agents de relations publiques, comme dans une entreprise privée. Jouissant d'une grande visibilité, ils sont rapidement devenus vedettes de la télévision et de la radio. Toujours revêtus de l'uniforme, ils animaient des émissions portant sur la prévention du crime et, d'une façon générale, sur le travail policier dans son ensemble. L'un d'eux, fort de cette popularité soudaine, s'est lancé en politique et est devenu député à Québec.

Malgré tout, à Montréal, quelques bons vieux journalistes du beat avaient résisté au raz de marée et perpétuaient la tradition. Ils avaient encore leurs bureaux à l'intérieur de la station de police principale. Ces journalistes avaient accès à toutes les sources d'information, à tous les bureaux et services, dans l'édifice de la police de Montréal. On aurait facilement pu les confondre avec les détectives dont ils étaient d'ailleurs les fidèles auxiliaires. Ces journalistes avaient une insigne et une carte d'identité presque en tous points semblables à celles des policiers, ce qui leur facilitait grandement la tâche, dans leur quête d'informations.

Cette relation journalistes-policiers était telle que les journalistes du beat puisaient directement dans les rapports quotidiens d'activités policières leurs sujets de nouvelles et de reportages. Chaque matin, arrivaient à la centrale de police, par courrier spécial, tous les rapports d'incidents enregistrés la veille dans les divers postes de la ville. Les journalistes ouvraient ce courrier et y choisissaient leurs nouvelles, souvent avant mème que les officiers responsables n'en aient pris connaissance. Ces messieurs téléphonaient ensuite à leurs chefs de nouvelles qui dépêchaient photographes et reporters sur les lieux. Se contentant généralement de donner leurs nouvelles par téléphone, ces journalistes du beat ne se déplaçaient que pour les «grosses» histoires de meurtre ou de hold-up, en compagnie des détectives spécialisés. Rien ne s'écrivait, il va sans dire, sans que le détective ne l'autorise et n'était publié que ce qui ne pouvait pas nuire à l'enquête.

Au fur et à mesure que ces vieux joumalistes ont pris leur retraite, personne n'a été autorisé à les remplacer et on a finalement fermé cette salle de presse, avec tous les privilèges qui y étaient rattachés.

Peu à peu, le service de relations publiques a été chargé de diffuser les nouvelles, ce qui a souvent provoqué des frictions entre relationnistes et certains groupes de détectives, qui tenaient à contrôler l'information et à privilégier les reporters "corrects et fidèles", et les agents de relations publiques, qui voulaient traiter tous les journalistes également. Ce vieux conflit demeure toujours latent et refait surface à tout moment, encore aujourd'hui. 
Traditionnellement, à Montréal comme dans toutes les grandes villes, le détective jouit d'un prestige qui lui donne des pouvoirs et une autonomie que lui envient ses collègues en uniforme. C'est ainsi que le détective peut entretenir avec les journalistes des relations suivies, plus personnelles, un peu comme il le fera avec des avocats, des informateurs, des gens du milieu, sans avoir à rendre compte.

Le détective utilise donc la presse, régulièrement, dans ses stratégies d'enquête. Les procédés sont classiques, on y pratique souvent davantage la désinformation que l'information: on laisse croire que le coupable est sur le point d'être démasqué alors qu'on veut surprendre le suspect; on visibilise l'arrestation des témoins récalcitrants, laissant entendre qu'ils ont parlé; on n'hésitera pas à cacher ou à fausser des renseignements afin de créer la diversion, provoquer suspects et témoins. Le joumaliste y trouve son compte puisque l'enquêteur lui donnera des informations privilégiées, lui fournira photos de victimes ou de suspects, lui permettra d'être le premier sur les lieux des événements importants. Le détective pratique donc avec les journalistes le même bargaining qu'avec les victimes, les témoins, les accusés. Certaines entrevues télévisées récentes avec des délateurs célèbres, concoctées par les policiers, relevaient davantage de la tactique policière que de l'information.

En principe, ces relations journalistes-policiers sont assez mal vues des autorités policières, mais l'enquêteur peut à peu près toujours les justifier en les situant dans sa stratégie, comme il peut le faire pour une rencontre avec un informateur, dans un bar. Il a beau jeu puisqu'il reconstruit littéralement les événements, après coup, et les légitime, tant dans ses rapports à la hiérarchie que par les médias.

Avec l'écoute électronique, les enquêteurs, utilisent plus que jamais les médias: on lance un message, une fausse nouvelle à des témoins, des suspects ou des complices, via les médias, et on attend les retombées. Il suffit de se mettre à l'écoute de chacun, les téléphones ne dérougissent pas. À la Sûreté du Québec, on en a appris davantage de cette façon sur les gangs de motards que par les voies traditionnelles d'enquête. Le même procédé avait réussi à démasquer le mafioso Vincente Cotroni et son clan, pendant les audiences de la Commission d'enquête sur le crime organisé (CECO), en 1975.

Peut-on aller jusqu'à affirmer que la police peut utiliser les médias non plus seulement afin de désinformer ou faire diversion mais jusqu'à légitimer des actions douteuses ou franchement condamnables, des gestes illégaux? C'est ce que soutient une étude, publiée en 1978 par l'Office des droits des détenus en annexe d'un rapport intitulé «Violence et presse 
écrite" (Gosselin et al., pp. I à XXXV). On y fait l'analyse systématique d'une série de guet-appens où des policiers ont abattu des voleurs de banque.

Le Comité des droits des détenus a voulu faire la preuve qu'il existait au Québec un véritable "escadron de la mort" et que, grâce aux enquêtes du coroner et aux médias, ces actions meurtrières étaient toujours et systématiquement légitimées!

Dans certains cas, la relation journalistes-policiers devient encore plus que particulière, l'un se mettant franchement au service de l'autre. C'est le summum de la complicité et de la conspiration police-médias. C'est le cas du journaliste Claude Jodoin, chroniqueur policier et judiciaire au Journal de Montréal, de 1965 à 1975, qui est devenu délateur officiel, après avoir infiltré un gang de criminels. Jodoin, de 1971 à 1982, travaillait pour "le clan des Dubois", un gang spécialisé dans le trafic de drogue, la protection et les "contrats" meurtriers.

En 1982, il passait du côté des policiers et, moyennant salaire et protection, dénonçait le gang des Dubois, d'abord devant la CECO puis devant les tribunaux. Fort de son statut privilégié, il a écrit depuis deux livres portant sur deux affaires criminelles. Pouvant compter sur les informations privilégiées que lui foumissent ses "patrons" policiers (Jodoin 1982 et 1984), Jodoin y diffuse intégralement le message policier.

La Commission Keable a déjà dénoncé l'action d'une autre informatrice policière, journaliste à la pige, Carole de Vault, mêlée très directement aux événements d'Octobre 70 , au Québec. Jouissant elle aussi des mêmes relations privilégiées que Jodoin, elle a déjà écrit deux livres (de Vault 1981 et 1984), elle aussi en pouvant compter sur d'excellentes sources d'informations. Après avoir travaillé avec et pour les policiers de l'Escouade antiterroriste, transformée depuis en Brigade antigang, avec à peu près les mêmes hommes et les mêmes méthodes, elle continue encore à écrire des articles, à collaborer étroitement avec les policiers et à fréquenter le Palais de justice de Montréal, à titre de journalistc à la pige.

La trop grande complicité entre détectives et chroniqueurs policiers peut aussi donner lieu à des relations pour le moins particulières. À la Sûreté du Québec, par exemple, certains policiers ont dû être transférés et affectés à d'autres fonctions, pour avoir entretenu des relations un peu trop suivies avec des journalistes. Dans certains cas, il devenait évident que le policier utilisait les médias pour se "visibiliser", se donner tout le mérite des enquêtes, afin de faire avancer sa carrière. Il y a quelques 
années, deux policiers, de la Section de l'identité judiciaire, à Montréal, étaient forcés de démissionner pour avoir un peu trop «collaboré» avec des journalistes. Les deux hommes, semble-t-il, touchaient des sommes d'argent chaque fois qu'ils appelaient certains journalistes pour les aviser qu'un crime majeur venait d'être commis. Ils auraient également vendu des photos de scènes de crimes et de dossiers à des journalistes.

À la police de Montréal, des détectives ont aussi été mutés pour avoir trop collaboré avec certains journalistes. Les membres d'une escouade complète ont été accusés, publiquement, dans un document remis au ministère de la Justice et à la presse, et rédigé par l'Office des droits des détenus, de trafic d'influences avec certains journalistes. (Gosselinet al., p. 38)

C'est donc en bonne partie pour mettre fin à ces relations particulières entre journalistes et détectives qu'un véritable service d'information, qu'on a continué d'appeler service de relations publiques, fut créé à la police de Montréal.

Les policiers relationnistes se sont familiarisés avec le métier de journaliste, ont suivi des cours, obtenant notamment un certificat en relations publiques de l'Université de Montréal. Cette formation professionnelle devait creuser encore davantage le fossé qui séparait les relationnistes des détectives. On a mené une lutte farouche aux policiersvedettes dont on vantait un peu trop les exploits dans les médias pour en arriver, systématiquement, à concentrer toute la diffusion de l'information entre les mains du service des relations publiques.

Certains conflits, semble-t-il, persisteront à ce sujet tant et aussi longtemps que se côtoieront, dans un corps de police, les détectives traditionnels et de jeunes professionnels. Ce conflit, d'ailleurs, a connu des moments de crise aiguë, notamment au cours des événements d'Octobre 70, alors que les membres du Service de relations publiques ont refusé de donner des cartes de presse à certains policiers qui voulaient infiltrer certaines réunions et manifestations.

À Montréal, donc, on ne retrouve pas de politique figée de relations police-médias. Il faut composer avec la réalité quotidienne et les politiques des administrations du moment.

Pas étonnant, alors, qu'on ne retrouve à peu près aucune directive formelle et écrite sur le sujet.

Dans le document «règlement sur la déontologie et la discipline des policiers de la Communauté urbaine de Montréal", on retrouve, à la Section 2, intitulée "Les devoirs de service public", à l'article 8: 
"Constitue notamment une faute disciplinaire

a) le fait d'accepter, de solliciter ou d'exiger, directement ou indirectement, un don, une promesse, une récompense, une commission, un rabais, un prêt, une remise de dette, une faveur ou tout autre avantage ou considération, de nature à nuire ou compromettre son impartialité dans l'accomplissement de ses fonctions.

c) le fait d'utiliser à des fins personnelles ou dans le but d'en tirer un avantage ou un profit, les informations obtenues à l'occasion de l'exercice de ses fonctions, ou à cause de sa situation dans le Service de la Police.

h) le fait de rechercher la notoriété, directement ou indirectement, par l'intermédiaire des médias d'information».

À la police de Montréal, le contrôle de l'information semble avoir toujours été un enjeu important.

Au début des années 70 , une guerre de pouvoir divisait la police de Montréal. Deux clans s'affrontaient: celui du directeur de la police, frère du président du Comité exécutif de Montréal; celui du Conseil de sécurité, qui entendait administrer à sa façon. Un clan utilisait la Presse et le journaliste Michel Auger dans sa stratégie. L'autre clan alimentait de nouvelles le Devoir et son journaliste Jean-Pierre Charbonneau. C'est ce dernier qui a eu le dernier mot en publiant, à la une du Devoir, que le directeur de police Jean-Jacques Saulnier avait accepté un poste de télévision en guise de pot-de-vin. Le clan Saulnier avait perdu la guerre, en bonne partie "grâce" à un journal.

Ce fut à nouveau le froid entre la police et les médias. Les relations officielles étaient rompues. L'information redevint la chose des enquêteurs qui renouèrent avec leur ancien système.

Par la suite, les politiques ont varié, d'une administration à une autre. Le directeur Jean-Paul Gilbert, un universitaire, a surtout voulu polir l'image de la police dans le grand public et particulièrement auprès des jeunes. Son successeur Saint-Aubin, un policier de la base, a coupé tous les ponts avec les journalistes. Comme tous les policiers issus de la patrouille, il avait une dent contre les journalistes. À Montréal, comme sans doute dans toutes les grandes villes, s'il y a complicité entre détectives et journalistes, une hostilité souvent farouche oppose patrouilleurs et journalistes. Ces agents en uniformes, ce sont les policiers dont les noms et les photos n'apparaissent pas dans le générique des belles enquêtes réussies mais que l'on voit au premier rang, au téléjournal 
ou dans une photo de première page, le lendemain d'une manifestation violente, matraque à la main.

Ce vieux conflit n'est pas près de s'éteindre. Le 10 mars courant, un caméraman de Radio-Canada était acquitté de voies de fait contre un policier, à Montréal. L'accrochage s'était produit sur les lieux d'une prise d'otage dont les policiers voulaient protéger le périmètre. Le 17 mars, ce caméraman intentait une poursuite de $95700 \$$ à titre de dommages contre la Communauté urbaine de Montréal et un de ses policiers. Un autre caméraman, de CFCF-TV, avec un technicien de la même station et un photographe de presse, ont poursuivi des policiers, qui les auraient molestés, au cours des manifestations qui ont marqué la soirée du référendum, en 1980. Après avoir obtenu gain de cause devant la Commission de police, ils ont entamé des poursuites au civil dont ils attendent les résultats.

Avec l'actuelle direction, on entend rouvrir les relations avec les médias, mais cette fois en décentralisant, surtout afin de rencontrer les exigences des coupures budgétaires projetées.

On étudie en ce moment la possibilité d'acheminer directement aux médias, sur télescripteurs, les nouvelles policières, comme on le fait à New York et dans plusieurs autres grandes villes américaines. On peut ainsi censurer l'information à la source et faire le tri dans la nouvelle.

Le service de relations publiques de la police de Montréal, avec trois agents, un sergent, un lieutenant et un directeur, ayant presque tous reçu une formation professionnelle spécialisée, resteront en poste mais ne détiendront plus le monopole de la communication des nouvelles. Des officiers de service seront autorisés à transiger avec les journalistes.

\section{À LA GENDARMERIE ROYALE: MÉFIANCE ET HOSTILITÉ}

Si les relations police-médias illustrent surtout la complicité à la police de Montréal, c'est l'hostilité et la méfiance qui marquent les relations, ou l'absence de relations, à la Gendarmerie royale. Le spectre des commissions d'enquête McDonald et Keable hantent encore la hiérarchie.

Il y a 10 ans, en mars 1976, l'ex-policier Robert Samson, à son procès pour avoir placé une bombe chez un important industriel de Montréal, déclarait qu'il avait posé des gestes beaucoup plus graves que ceux-là en tant que membre de la Gendarmerie royale du Canada. Les journalistes se sont vite emparés de cette fuite et les événements se sont précipités. Sans doute pour ne pas être en reste avec leurs collègues 
américains, qui «profitaient» de Watergate, les joumalistes se sont fait l'écho fidèle, des mois durant, de toutes les critiques dirigées contre la Gendarmerie royale et de ses actions subversives et illégales. L'émission "Fifth Estate", qui a particulièrement bien couvert ce dossier, a valu à $\mathrm{CBC}$ des critiques et menaces de porte-parole du gouvernement fédéral qui voyaient là un danger pour la sécurité nationale.

Jusqu'alors, ce ne fut jamais la plus étroite et chaleureuse collaboration entre policiers de la Gendarmerie royale et journalistes, au Québec, mais les relations étaient cordiales. Le service des relations publiques de la Gendarmerie royale collaborait avec les journalistes qui désiraient faire des features sur les diverses activités des policiers fédéraux. On faisait parvenir régulièrement des communiqués de presse aux médias, dans des campagnes de prévention et de sécurité. Régulièrement, on invitait les médias à la suite d'une spectaculaire saisie, laissant les reporters photographier la drogue ou les faux billets saisis et même quelques officiers supérieurs qu'on pouvait interviewer. Les médias étaient même parfois invités à accompagner les policiers lors de certaines descentes, surtout dans les années 70 . On jugeait alors important de visualiser l'action des policiers fédéraux au Québec, surtout qu'avec l'avènement du Parti québécois, il était de plus en plus question d'une police nationale au Québec. Même les libéraux de Robert Bourassa avec le ministre Jérôme Choquette voulaient réclamer d'Ottawa un remboursement de plusieurs millions pour compenser les frais encourus par notre propre police provinciale. Avec l'avènement d'un gouvernement péquiste, en 1976, il était impérieux de faire la preuve de l'utilité des policiers fédéraux au Québec. Il y avait d'ailleurs à Montréal, en 1977, cinq policiers attachés au service des relations publiques, à la Gendarmerie royale.

Dix ans plus tard, le service de relations publiques n'existe plus. Seul un policier, maintenant devenu «coordonnateur de la Prévention du crime et des relations socio-policières" peut traiter avec les médias, le plus rarement possible, à pas feutrés et sur des sujets neutres et sans conséquences possibles.

Il suffit d'entrer dans l'édifice de la Gendarmerie royale, à Montréal, pour saisir rapidement la situation. Un journaliste ne peut circuler dans l'immeuble. Il doit s'identifier formellement, surveillé comme les autres visiteurs par un réseau de télévision en circuit fermé. Il doit avoir un rendez-vous préalable et ne peut qu' accompagner, dans son bureau, le policier avec qui il a rendez-vous. Celui-ci répond de lui et doit venir le reconduire à la sortie, l'entrevue terminée, après avoir pris grand soin de bien enregistrer l'heure d'arrivée et de départ. 
Dans un manuel d'administration daté de décembre 1986, au chapitre III.2, voici comment se traduisent, à la Gendarmerie royale, les relations police-médias (reproduction intégrale du document):

\section{RENSEIGNEMENTS À FOURNIR À LA PRESSE}

D.I GÉNÉRALITÉS

D. 1.a. Il est interdit de révéler aux médias d'information ou à un journaliste un renseignement qui ne doit pas être diffusé par la presse, à savoir:

1. Nom ou identité de la personne sous arrestation ou accusée, avant la comparution devant le tribunal.

2. L'envergure, la durée, les modalités d'enquête et la mise en accusation.

D.1.b. La Presse ne doit pas être invitée à photographier les lieux, objets saisis et/ou l'exhibition de pièces à conviction potentielles qui pourraient être préjudiciables aux accusés.

\section{2 CONFÉRENCE DE PRESSE}

D.2.a. Lorsqu'il est effectivement nécessaire de tenir une conférence de presse:

1. Utiliser une déclaration préparée à l'avance répondant aux questions élémentaires (qui? quoi? où? quand? et pourquoi?).

2. Obtenir l'approbation de l'Officier commandant sous-divisionnaire et transmettre le contenu du communiqué via CIPC à la D.G., la sous-direction concernée à l'attention des Relations publiques.

3. Remettre le communiqué au journaliste à son arrivée.

4. Aménager la salle ou lieu de conférence en conséquence.

5. Assurer la sécurité dans l'édifice. (À Montréal aviser la réception à la section de la Sécurité de l'Édifice.)

\section{À LA SÛRETÉ DU QUÉBEC: COMMUNICATION = STRATÉGIE ET CONTRÔLE}

À la Sûreté du Québec, ce sont les événements d'Octobre 70 qui ont forcé les autorités à mettre au point une véritable politique de communications avec la presse. Jusque-là, c'était la pagaille. 
Les journalistes circulaient dans l'édifice de la Sûreté du Québec, à Montréal, comme dans un moulin. Ils transigeaient directement avec les policiers de leur choix. Les détectives-vedettes se bâtissaient littéralement des carrières en se faisant très visibles dans les médias. Des cartes de presse, portant la photo et les empreintes digitales de leurs détenteurs, avaient été émises par la Sûreté du Québec. Ces «dossiers» étaient classés, au Service de l'identité judiciaire, par ordre alphabétique, au même titre et au même endroit que les dossiers judiciaires des criminels condamnés. Ces cartes avaient été distribuées si généreusement que des dizaines de sympathisants et participants directs aux événements violents d'Octobre 70 en étaient détenteurs. À la police de Montréal aussi, on avait aussi émis une carte à peu près semblable. Le Conseil de presse avait recommandé qu'on fasse disparaître ces cartes, à la suite de plaintes de journalistes, travaillant dans des publications syndicales et plus engagées, qui se disaient harcelés par les policiers. Certains journalistes ont été arrêtés pour des contraventions impayées parce qu'on les avait retracés grâce à leur carte de presse.

Une carte, avec photo, est maintenant émise au nom des journalistes qui en font la demande, afin de leur permettre de circuler dans l'édifice de la Sûreté du Québec à Montréal mais cette carte ne sort pas de l'immeuble et n'est utilisée que là. Elle est classée au centre d'accueil de l'immeuble, où les journalistes ne peuvent même plus se rendre à l'ascenseur sans être interceptés.

De 1968 à 1970, il y a bien eu un service de relations publiques à la Sûreté du Québec, mais on n'y faisait aucune diffusion de nouvelles. Plus souvent qu'autrement, ces policiers servaient de chauffeurs ou de commissionnaires spéciaux pour ces messieurs de la haute direction.

En 1971, un civil, fonctionnaire du ministère des Communications et ex-directeur de l'information et de la publicité de l'Office de l'information et de la publicité du Québec à Montréal, entrait en scène pour faire le grand ménage. C'était la veille de la première grève des policiers. Il avait pour mandat de créer un véritable service de communication.

Dix ans plus tard, avec 15 policiers à Montréal et neuf en province, le Service de l'information peut maintenant se vanter de "contrôler" l'information.

Tout d'abord, aucun journaliste ne peut maintenant prendre directement connaissance des nouvelles qui arrivent au quartier général de Montréal, de tous les coins de la province, par téléscripteur. Les agents du service des communications font le tri de ces nouvelles et ne diffusent que ce qu'ils jugent susceptibles d'être diffusé. Dans les affaires plus 
importantes, on émettra un communiqué succinct, squelettique. Par la suite, on permettra à certains journalistes d'en apprendre davantage, selon le média, le journaliste, les circonstances et la stratégie.

Même le commandant ne peut annoncer une nouvelle sans avoir préalablement consulté le Service des Communications. Les entrevues données par le directeur et ses principaux lieutenants sont rarissimes. On refuse systématiquement de commenter l'actualité, sauf dans le cadre d'une stratégie bien définie.

Tout a été prévu pour que le Service de l'information jouisse d'une autonomie presque totale. Il ne relève ni ne dépend de la hiérarchie normale mais directement du directeur du personnel. C'est ainsi que le directeur de l'information n'a d'ordre à recevoir d'à peu près personne et peut imposer ses politiques à tous les directeurs de sections ou de services. C'est ainsi également qu'il est étroitement mêlé aux grandes enquêtes criminelles, mettant l'information au service des stratégies, comme instrument et méthode d'enquête.

Cette conception des communications au service de la stratégie policière peut facilement se traduire par la manipulation des médias. Un exemple, qui a fait beaucoup parler, dans tout le Québec, mérite d'être cité:

C'était en 1977. Le gérant de crédit d'une succursale des Caisses Desjardins, à Sherbrooke, avait été kidnappé. Une rançon de 1 million \$ était exigée. L'enquête piétinait. Des policiers étaient convaincus que l'homme avait organisé son propre enlèvement, afin de soutirer la forte somme à son employeur, davantage dans un but de vengeance. Dans leur stratégie en vue de démasquer l'employé de banque, les policiers ont habilement utilisé les médias, "confiant» aux journalistes leur thèse... évidemment sous le sceau de la confidence. À peu près tous les journalistes sont tombés dans le panneau et deux médias ont dépassé les limites. Un hebdo a sommé carrément la victime Charles Marion de sortir de sa cachette. Radio-Canada, dans son téléjournal de $18 \mathrm{~h}$, a parlé ouvertement de supercherie et de fumisterie... 83 jours après le kidnapping, Charles Marion était retrouvé et on établissait hors de tout doute qu'il avait été bel et bien victime d'un véritable enlèvement. Les deux médias furent poursuivis pour $125000 \$$ chacun. Dans les deux cas, un règlement hors-cours est intervenu. Les policiers n'avaient pas intérêt à ce que tous les dessous de cette affaire ne se sachent et ils ont joué toutes les cordes pour éviter le procès. Les policiers étaient d'ailleurs tellement convaincus qu'il s'agissait d'une supercherie avant le dénouement de l'affaire, qu'ils avaient utilisé deux journalistes, comme émissaires, pour 
aller remettre la rançon à ceux qu'ils croyaient être de faux kidnappeurs. À leur insu, les journalistes Claude Poirier et Normand Maltais avaient livré deux valises remplies de coupures de journaux, au lieu des $500000 \$$ promis, ce qui aurait pu leur être funeste et sérieusement mettre en danger la vie de la victime. Cette fois, les journalistes ont été manipulés à leur insu, croyant transporter d'authentiques billets de banque!

On se défend bien de pratiquer de la désinformation à la Sûreté du Québec, comme de faire des relations publiques avec des événements criminels mais certains cas récents soulèvent de sérieuses questions à ce sujet. Entre autres, celui des Hell's Angels, ce gang de motards accusés d’avoir liquidé six de leurs membres dans une tuerie dont tous les médias nous ont gavés d'images depuis juin 1985.

Pendant l'enquête du coroner, qui s'est prolongée sur une période de plus de trois mois, à Joliette, les journalistes ont eu droit à tous les petits soins, de la part du service des communications de la Sûreté du Québec. (Il importe de rappeler que cette enquête n'avait pas été confiée au coroner du district judiciaire qui normalement aurait dû la mener mais à un juge, nommé coroner ad hoc par le ministre de la Justice.) Pendant toute cette enquête du coroner, des agents du service des communications de la Sûreté du Québec étaient à la disposition des journalistes pour leur fournir informations et photographies. On avait même mis à leur disposition une maison mobile, leur servant de salle de presse et dotée de toute la quincaillerie technique nécessaire aux journalistes de la presse électronique et écrite. Jamais, de mémoire de journaliste, un tel déploiement n'a été fait au Québec à l'occasion d'une affaire policière et judiciaire. La collection complète des photos couleurs de tous les "suspects" était à la disposition des médias.

Jour après jour, photographes et caméramen étaient là, au moment où une bonne vingtaine de motards, pieds et poignets enchaînés, paradaient, entourés de dizaines de policiers, déguisés en uniformes de combat, comme les héros de certaines séries de télévision américaine.

Il n'est pas mauvais de rappeler qu' avant ce scénario, la concurrence avait été très forte, entre nos trois grands corps policiers, au sujet de ce dossier. C'est d'abord la Gendarmerie royale qui a été mise sur la piste de cette affaire par le FBI. La police de Montréal avait déjà un volumineux dossier sur le sujet et il ne lui manquait plus que les budgets pour faire avancer les choses. "L'achat" des informateurs-délateurs et l'écoute électronique représentaient de fortes sommes qui ont été refusées aux policiers de Montréal. 
À la Sûreté du Québec, des budgets faramineux ont été consacrés et le sont encore à ce dossier. Cette survisualisation de l'action et de l'efficacité de la Sûreté du Québec, a-t-on expliqué officiellement, se situent dans le cadre de la stratégie policière, afin d'éliminer, de «brûler", chaque membre du gang... Malgré tout, cette opération arrivait à point nommé:

Nous étions alors en juin 1985 et c'est précisément à ce moment que la Régie de l'assurance automobile du Québec dévoilait des statistiques fort compromettantes pour la Sûreté du Québec. Dans son rapport annuel, la Régie révélait qu'on avait enregistré 33934 accidents avec dommages corporels sur les routes du Québec en 1982; 36861 en 1983 et 41108 en 1984. Par ailleurs, les policiers de la Sûreté du Québec avaient distribué 426962 contraventions en 1982, 410073 en 1983 et 265831 en $1984 \ldots$ Le nombre de contraventions étant inversement proportionnel au nombre d'accidents, la direction de la Régie de l'assurance automobile a accusé les policiers d'être les grands responsables de cette augmentation des accidents graves. L'accusation a été très visualisée car elle arrivait peu de temps après une grève de zèle à la Sûreté du Québec qui avait choqué l'opinion.

Autre «coïncidence»: pendant tout ce temps, le ministre de la Justice, alors grand patron de la Sûreté du Québec, menait sa campagne à la chefferie du Parti québécois. À peine cette campagne terminée, le ministre de la Justice était plongé dans la campagne électorale en vue des élections générales de 1985. Avait-il le choix de refuser les budgets spéciaux réclamés par ses policiers?

Les policiers nous ont présenté, dans les médias, le feuilleton des faits et gestes des délateurs dont ils avaient acheté les services, survisualisant l'événement. Un tueur à gage converti qui vient avouer 12 homicides «involontaires», un autre qui vient en confesser 43 , ce n'est certes pas le meilleur exemple de cohérence et de justice pour un système judiciaire, mais une excellente opération de marketing pour un corps policier... et un ministre de la Justice!

Plus d'un an après le début de cette affaire, quelques semaines avant que ne commence le procès pour meurtre de quatre membres du gang des Hell's Angels, à Montréal, le magazine de la Sûreté du Québec, "Sûreté" (août 1986, $\mathrm{n}^{\circ} 7$ ), consacrait presque toute son édition (26 pages sur 32) aux bandes de motards et plus particulièrement aux Hell's Angels, le tout appuyé de plus de trente illustrations dignes des feuilles les plus croustillantes. Le journal La Presse a repris à la une ce reportage, même s'il en avait déjà publié toutes les informations, un an plus tôt. 
L'officier responsable des enquêtes criminelles à la Sûreté du Québec, le directeur des communications et la rédactrice en chef de la revue ont tous été cités pour outrage au tribunal, les deux premiers formant "le comité d'orientation" du magazine.

Malgré tout, les arguments en faveur de la nécessité du contrôle de l'information ne manquent pas, à la Sûreté du Québec, surtout depuis l'avènement de la Commission et de la Charte des droits de la personne alors que la Sûreté est régulièrement poursuivie pour avoir diffusé certaines nouvelles.

Une poursuite de 1,5 million $\$$ est venue renforcer cette politique de contrôle de l'information. Elle a été intentée par un haut fonctionnaire, chef du contentieux au ministère de la Justice, à Québec. L'avocat poursuit le gouvernement du Québec pour avoir sali sa réputation. On avait divulgué, en 1982, qu'on menait une enquête sur lui et cette nouvelle n'avait pas été diffusée par le Service des communications. L'improvisation et l'amateurisme qui ont marqué les relations des policiers avec les médias, au moment des événements d'Octobre 70, servent encore de justification et de légitimation à ce contrôle et à cette manipulation de l'information.

Ce parfait contrôle a pu être mis à l'épreuve en 1984 alors que pendant des mois les policiers de la Sûreté du Québec ont enquêté sur une demande de rançon faite par un individu qui prétendait avoir «empoisonné" un certain médicament fabriqué par la compagnie Parke-Davis, une multinationale de Détroit. Quatre fois la rançon fut versée mais jamais l'homme n'a été démasqué. La compagnie a retiré le médicament des tablettes de toutes les pharmacies, de Halifax à Vancouver, de même qu'en Nouvelle Angleterre. Peut-être a-t-on eu raison, à la Sûreté du Québec, de ne pas dévoiler la chose mais c'est là un tour de force peu banal que d'avoir réussi à cacher cette nouvelle à tous les médias alors qu'on était partout plongé dans la psychose du Tylenol!

Les directives officielles, émises en 1977 et modifiées le 15 octobre 1985, sont fort éloquentes sur ce sujet. En voici quelques extraits:

«Diffusion d'informations

aux masse-médias lors d'évé-

nements d'importance

\section{PRINCIPES GÉNÉRAUX}

2.1 La diffusion d'informations aux masse-médias est une fonction centralisée, contrôlée par le Service des communications qui a la responsabilité des moyens utilisés. 
2.2 Lors d'un événement d'importance, seul le Service des communications et le personnel qu'il désigne, sont habilités à diffuser de l'information aux masse-médias.

2.4 Nonobstant la collaboration entre la Sûreté du Québec et les massemédias et même si la Sûreté du Québec est un organisme public, aucune diffusion de l'information ne peut s'effectuer lorsque la divulgation de communications privilégiées est déjà restreinte par la directive DOSS. OPER. -05 et/ou peut: (...)

\section{RÔLES DE L'AGENT D'INFORMATION}

Suivant les instructions du Service des communications:

6.l Prépare, conjointement avec le responsable d'unité et le membre assigné à l'événement, la diffusion d'informations aux masse-médias;

6.2 Se charge, sur les lieux, de regrouper et de contrôler les représentants des masse-médias;

6.3 Participe à la diffusion des informations générales aptes à être diffusées dans l'immédiat;

6.4 Fournit, autant que possible, les motifs d'un refus de divulguer certaines informations (...) afin d'obtenir la compréhension des masse-médias et de conserver leur collaboration;

\section{RÔLE DU SERVICE DES COMMUNICATIONS}

7.1 Contrôle la diffusion de l'information aux masse-médias.

7.2 Si nécessaire, intervient pour:

7.2.I décider de la pertinence d'une diffusion ou de la pertinence de diffuser certaines informations;

7.2.2 modifier le processus de diffusion et/ou

7.2.3 faire cesser la diffusion d'informations.

7.3 Lorsque approprié, autorise expressément une personne à diffuser de l'information aux masse-médias.

7.4 Évalue les conséquences des divulgations non autorisées et formule les recommandations appropriées."

Le contrôle absolu et centralisé de l'information est évident, à la Sûreté du Québec, mais il s'agit là d'une politique officielle, écrite et affichée. 


\section{CONCLUSION}

À la lumière de la situation actuelle, au Québec, les conflits idéologiques entre journalistes et policiers ne sont que littérature. Le rapport de force et la tension qui devraient régler les relations entre les deux groupes ne sont plus là. L'apathie complice des journalistes l'emporte sur la méfiance, la réticence et l'hostilité qui devraient marquer l'interrelation médias-police. Cette apathie, on ne sait plus très bien si elle est faite d'ignorance et d'indifférence, ou feinte afin de faire oublier le traitement de l'information comme produit de consommation éminemment rentable.

Le pouvoir d'informer, ce ne sont plus les journalistes mais les policiers qui l'exercent, du moins dans le champ d'action qu'ils ont en commun. Les journalistes ont troqué leur pouvoir pour leur pitance quotidienne de romans faciles, peu coûteux et qui se vendent bien. L'information policière est tronquée, partiale et charrie les stéréotypes qui desservent le mieux les policiers. Les joumalistes devraient cesser de rêver à Watergate et au journalisme d'enquête et pratiquer du journalisme d'information plus critique, en diversifiant leurs sources. Un reportage n'est pas un rapport de police. Les journalistes devraient se distancer des policiers et mener leurs propres enquêtes, cueillant l'information à toutes les sources possibles et, surtout, aller au-delà de l'événement pour le contextualiser, le situer dans l'activité et le processus de la société dans son ensemble.

Il faudrait, pour ce faire, que le journaliste puisse avoir accès directement à l'information, sans la censure policière et le filtrage des communicateurs professionnels. La chronique policière et du fait divers est trop lourde de conséquences, dans la masse de la clientèle captive des médias, pour qu'elle soit à ce point négligée. Un code d'éthique éviterait aux journalistes de commettre les injustices qui légitiment trop souvent les institutions de contrôle à censurer l'information. La syndicalisation avait suscité des espoirs en ce sens qui n'ont jamais été comblés.

Les policiers ne cèderont certes pas d'eux-mêmes ce pouvoir sur l'information qu'ils se sont arrogé, il sert trop bien leurs intérêts. Les journalistes sont seuls capables de corriger la situation.

En attendant, force nous est d'admettre que nous voguons allègrement vers une justice secrète, à l'abri des indiscrétions des médias.

Un incident se produit, les policiers font diffuser «leur" version des faits. La comparution se fait par une simple lecture de l'acte d'accusation, l'enquête préliminaire se déroule à huis-clos et, quelques mois plus tard, 
le plea bargaining remplace le procès. Les journalistes seront peut-être là pour enregistrer la sentence mais le grand public ne saura jamais ce qui a valu cette sentence à l'accusé. Justice de cachets, retour aux châtiments sur le parvis de Notre-Dame alors que la foule applaudissait au spectacle du supplice ou de la mise à mort sans savoir pourquoi et comment le supplicié avait été condamné.

C'est un aspect de la question. L'autre, c'est cet actuel mouvement de droite, de répression, qui prend du terrain, de jour en jour, sous les yeux d'une opinion complaisante. Et cette opinion est souvent acquise par la perception qu'elle a de la menace criminelle que lui présentent les policiers à travers les médias d'information.

Le vieux débat sur les journaux dits à sensations, les journaux jaunes ou les penny journals est usé et totalement dépassé. C'est maintenant la conception du fait divers, légitimé par une certaine idéologie et génératrice de nouvelles formes de contrôle social, qui devrait être sérieusement analysée.

\section{BIBLIOGRAPHIE}

ANDRÉ, J.D. (1979), "Presse et justice pénale: des fantasmes à ta réalité ", Déviance et Société ; vol. $3, \mathrm{n}^{\circ} 3, \mathrm{pp} .201$ à 227.

ALTSCHULL, J.H. (1975), "The Press and the Police: News Flow as Ideology", Journaly of Police Science and Administration, 3, 425-433.

CHRISTIENSEN, Jon, Janet SCHMIDT et Joel HENDERSON, (1982), "The Selling of the Police: Media, Ideology, and Crime Control", Contemporary Crises, vol. $6, n^{\circ} 3$, juillet, pp. 227 à 240 .

DA VIS, F. (1952), "Crime New in Colorado Newspapers", American Journal of Sociology, 57, pp. 325 à 330 .

FISHMAN, M., (1978), "Crimes Waves as Ideology", Social Problems, 26, pp. 113 à 126.

FOURNIER, F. (1982), F.L.Q. Histoire d'un mouvement clandestin, Éditions QuébecAmérique. Montréal.

GOSSELIN, L., E. MC FADDEN, M.L. PILON, TOUCHETTE, (1978), Violence et presse écrite, L'Office des droits des détenus de la Ligue des droits de l'homme.

HUMPHRIES, D. (1981), "Serious Crime, News Coverage, and Ideology", Crime and Detinquency, 27, pp. 191 à 212.

JODOIN, C. (1983), Mes aveux sur le clan Dubois, Éditions Québecor, Montréal.

JODOIN, C. (1984), Qui a tué Frank Shooffey?, Les Éditions de l'époque, Montréal.

POVEDA, T.G. (1982), "The FBI and Domestic Intelligence: Technocratic or Public Relations Triumph?, Crime and Delinquency, vol. 28, $n^{\circ} 7$, pp. 194 à 210. 
SELKE, W.L. et G.M. BARTOSZEK, (1984), «Police and the Media Relations: the Seed of Conflict», Criminal Justice Review, vol. $\mathrm{n}^{\circ} 2$, pp. 25 à 30 .

SMAUS, G. (1983) «Actualités bibliographiques: masse-médias et criminalité: état de la recherche en Allemagne et en Autriche", Déviance et Société; vol. 7, $n^{\circ} 3, p p .249$ à 263.

VAULT, C. (de) (1981), Toute ma vérité, Montréal, Stanké.

VAULT, C. (de) et R. DESMARAIS (1984), Donald Lavoie tueur à gages, Québec Livres. 